ISSN: $1130-3743$

\title{
CUATRO DÉCADAS DE TEORÍA DE LA EDUCACIÓN: ¿UNA ECUACIÓN IMPOSIBLE?
}

Four decades of Educational Theory. An impossible equation?

\section{Quatre décennies de Théorie de l'Éducation. Une équation impossible?}

\author{
Gonzalo Jover y Bianca THOILLIEz \\ Universidad Complutense de Madrid. Facultad de Educación. Departamento de \\ Teoría e Historia de la Educación. C/ Rector Royo Villanova, s/n. 28040 Madrid. \\ Correo-e: gjover@edu.ucm.esybthoilliez@edu.ucm.es
}

Fecha de recepción: enero de 2010

Fecha de aceptación definitiva: abril de 2010

Biblid [(1130-3743) 22, 1-2010, 43-64]

RESUMEN

Este artículo aborda el modo en que en las últimas cuatro décadas se ha configurado la Teoría de la Educación en España. Para ello, los autores utilizan, a modo de metáfora, el concepto de "ecuación", y presentan una serie de entrevistas realizadas a tres personas que han tenido un papel fundamental en el desarrollo del conocimiento teórico de la educación: Gaston Mialaret, Paul H. Hirst y Wilfred Carr. La "ecuación" está compuesta de dos valores conocidos y de una incógnita. Los valores son el referente francés de las ciencias de la educación y el referente británico de las disciplinas aplicadas a la educación. La incógnita a despejar es la propia Teoría de la Educación. El artículo señala que la Teoría de la Educación que se ha venido desarrollando en nuestro contexto ha tratado de aunar elementos heterogéneos desgajados de sus tradiciones de origen, concluyendo que para entender la ecuación de la educación lo que se necesita hoy es una teoría más centrada en investigar la educación misma. 
Palabras clave: Teoría de la Educación, epistemología educativa, ciencias de la educación, disciplinas aplicadas a la educación, Gaston Mialaret, Paul H. Hirst, Wilfred Carr.

SUMMARY

This article discusses how Spanish Educational Theory has been configured in the last four decades. To this end the authors use as a metaphor the concept of "equation" and present a series of interviews with three persons who have played a fundamental role in the development of theoretical knowledge of education: Gaston Mialaret, Paul H. Hirst and Wilfred Carr. The "equation" is composed of two known values and an unknown quantity, where the former are, on the one hand, the French component of educational sciences and, on the other, the UK factor of the disciplines applied to education; the unknown quantity to resolve is Theory of Education. The article argues that Theory of Education developed in our context has tried to unite heterogeneous elements isolated from their original traditions, and, however, what is needed today to understand the equation of education is a theory more focused on investigating education itself.

Key words: Theory of Education, educational epistemology, educational sciences, disciplines applied to education, Gaston Mialaret, Paul H. Hirst, Wilfred Carr.

\section{SOMMAIRE}

Cet article explique comment le cours des quatre dernières décennies a façonné la Théorie de l'Éducation en Espagne. Pour cela, les auteurs usent comme une métaphore la notion "d'équation" et ils présentent une série d'entretiens avec trois personnes qui ont joué un rôle déterminant dans le développement de la réflexion théorique de l'éducation: Gaston Mialaret, Paul H. Hirst et Wilfred Carr. "L'équation" est composé de deux valeurs connues et d'un inconnue, où les premiers sont, d'une part, la référence française des sciences de l'éducation et, d'autre part, la référence britannique des disciplines appliquées à l'éducation; l'inconnue à dégager est la Théorie de l'Éducation. L'article soutient que la Théorie de l'Éducation développée dans notre contexte, a tenté de réunir des éléments hétérogènes isolées de leurs traditions originales, et que, toutefois, pour comprendre l'équation de l'éducation, ce qui est nécessaire aujourd'hui c'est une théorie plus occupée à examiner l'éducation elle-même.

Mots clés: Théorie de l'Éducation, épistémologie éducative, sciences de l'éducation, disciplines appliquées à l'éducation, Gaston Mialaret, Paul H. Hirst, Wilfred Carr. 


\section{INTRODUCCIÓN}

Acudir a conceptos matemáticos para titular un trabajo que pretende abordar la configuración de la Teoría de la Educación en España puede parecer, en principio, algo extraño. Pero sólo en principio. Puede ser que ciertos problemas matemáticos y nuestro ámbito de conocimiento tengan, después de todo, más que ver entre sí de lo que cabría esperar.

Hace ahora cuarenta años, en 1969, se aprobó en la Facultad de Filosofía de la Universidad de Barcelona un plan de estudios novedoso, el llamado Plan Maluquer. Éste introducía, por primera vez en los estudios de Pedagogía, la asignatura de Teoría de la Educación que, junto con la de Introducción a la Pedagogía, venía a ocupar el hueco dejado por la Pedagogía General, denominación que se abandona. Se iniciaba con ello una tendencia que se generalizará poco después en otras universidades, con la sustitución, en la estructura organizativa de las Facultades de Filosofía y en el nombre de la titulación, del viejo nombre de pedagogía por el moderno de ciencias de la educación.

En 1972, con ocasión de la publicación de la Teoría de la educación de Juan Tusquets, Emilio Redondo se refería esta nueva situación señalando:

Desde que Herbart abordó en serio y por vez primera la empresa de hacer de la pedagogía una ciencia que descansara sobre el doble soporte de la ética y de la psicología, el tronco del saber pedagógico se ha ido engrosando de un modo espectacularmente rápido. Las abundantes ramas que de este tronco han surgido, junto con las aportaciones de otras muchas ciencias limítrofes que se han interesado por el tema de la educación, nos ofrecen hoy una panorámica tan abigarrada y compleja, que la arquitectura de la ya vieja "pedagogía general", montada sobre los supuestos herbartianos, es difícilmente reconocible. Hasta la misma palabra, de honda raigambre en los países latinos - -pedagogía - está hoy cediendo el paso a fórmulas plurales como la de "ciencias de la educación" que, hasta el presente, es la que ha tenido más fortuna. Cabría preguntarse si la pedagogía se nos habrá perdido en ese bosque de las ciencias de la educación. En todo caso, está hoy justificada esta otra pregunta ¿tiene todavía sentido hablar de una pedagogía general? (Redondo, 1972, 9).

Al hilo de la publicación del libro de Tusquets, Redondo planteaba, así, en clave de incógnita, el futuro de lo que hasta ese momento había sido la concepción del conocimiento teórico de la educación, inspirada en la tradición alemana, representada en Herbart, de un conocimiento unitario y general de raíz filosófica y vocación normativa (Schriewer, 2000).

¿Por qué en este contexto hablamos de "ecuación imposible»? Todo el mundo sabe que una ecuación se define como la igualdad entre dos expresiones algebraicas, en las que tenemos valores conocidos (datos) y desconocidos (incógnitas) que se relacionan entre sí a través de operaciones matemáticas. Para resolver una ecuación lo que tenemos que hacer es hallar los valores de las incógnitas que cumplan la igualdad planteada. Se sabe también que cualquier problema matemático puede 
ser expresado bajo la forma de una ecuación o de varias. Sin embargo, no todas las ecuaciones tienen solución. Puede ocurrir que haya más de un valor o, incluso, conjuntos de valores infinitos que satisfagan ese requisito. También puede suceder que no exista ningún valor de la incógnita que haga cierta una igualdad dada. Pues bien, pensamos que la forma en la que se ha desarrollado la construcción de la Teoría de la Educación en nuestro país a lo largo de las últimas cuatro décadas puede ponerse en clave de una ecuación imposible.

La historia del llamado último teorema de Fermat puede ilustrarnos en este punto. Pierre de Fermat nació en 1601, en Beaumont-de-Lomagne, al suroeste de Francia. Tras realizar sus estudios de Leyes en la Universidad de Toulouse, ocupó diversos cargos en la Administración y dedicó su tiempo libre a una ocupación por la que pasaría a la historia: las matemáticas. No publicó en su vida ningún libro, por lo que sus aportaciones las conocemos gracias a la correspondencia mantenida con otros matemáticos y a su afición a dejar anotaciones en los márgenes de los libros que leía. Su mayor inspirador fue Diofanto, de quien en 1621 aparecieron en Francia, traducidos al latín, seis de los trece libros de que constaba su Aritmética. Fermat hizo diversas anotaciones en los márgenes de esta edición. Tras su muerte, su hijo recopiló estos apuntes en la edición más famosa de la Aritmética de Diofanto, que incorporaba cuarenta y ocho anotaciones de Fermat. Fue una de estas anotaciones relacionada con el teorema de Pitágoras lo que hizo pasar a Fermat a la historia de las matemáticas (Torrecillas Jover, 1999, 11-22).

Los pitagóricos demostraron que en todo triángulo rectángulo el cuadrado de la hipotenusa es igual que el cuadrado de la suma de los catetos. Ahora bien ¿qué pasa si en lugar de cuadrados hablamos de cubos o potencias a la cuarta o la quinta? Fermat declaró que aunque todos los matemáticos del mundo dedicaran una eternidad a buscar solución a ecuaciones de la forma $\mathrm{x}^{\mathrm{n}}+\mathrm{y}^{\mathrm{n}}=\mathrm{z}^{\mathrm{n}}$, siempre que $\mathrm{n}$ fuese mayor que 2, jamás la encontrarían, por una razón muy simple: no existe. Es una ecuación imposible. En una de las anotaciones que hizo en su ejemplar de la Aritmética de Diofanto, lo dejó así escrito: "Es imposible dividir un cubo en suma de otros dos o un bicuadrado en otros dos bicuadrados, en general una potencia cualquiera superior a dos en dos potencias del mismo grado; he descubierto una demostración verdaderamente maravillosa pero este margen es demasiado estrecho para contenerla" (ibid., 90).

La explicación que aportaremos del modo en que, en los últimos cuarenta años, se ha venido configurando la disciplina de la Teoría de la Educación en la universidad española no igualará desde luego la audacia de este "aficionado" a las matemáticas. Pero la conclusión a la que llegaremos será, en cierto sentido, similar. Para empezar, baste con indicar que nuestra "ecuación" se compone también de dos valores conocidos y de una incógnita. Los primeros son dos perspectivas epistemológicas acerca del conocimiento de la educación, el referente francés de las ciencias de la educación y el referente británico de las disciplinas aplicadas a 
la educación ${ }^{1}$. La incógnita es la Teoría de la Educación que pretende fraguarse en nuestro país a partir de la combinación de esas dos perspectivas.

Dedicaremos las dos primeras partes del trabajo a conocer los datos de que se compone cada uno de los dos valores que entran en juego, para finalizar con las conclusiones en las que procuraremos "despejar la incógnita" de nuestra particular "ecuación imposible". Los datos de que se compone cada uno de los dos valores los hemos obtenido, además de las fuentes de documentación habituales, de varias entrevistas que realizamos a tres personas que han tenido a lo largo de estos últimos cuarenta años un papel fundamental en el desarrollo de conocimiento teórico de la educación en Europa, y que por diferentes vías han dejado su huella en lo acontecido en España: Gaston Mialaret (París, agosto de 2008), Paul H. Hirst (Londres, agosto de 2009) y Wilfred Carr (Chesterfield, agosto de 2009)².

\section{EL REFERENTE FRANCÉS}

El siete de diciembre de 1966, el Ministerio de Educación Nacional francés convocó a un reducido número de personas a una comisión, encargada de analizar el proyecto de creación de unos estudios dedicados a la educación, en el segundo ciclo de la formación universitaria. A la sesión asistieron Maurice Debesse, Gaston Mialaret y Paul Fraisse, junto a algunos representantes de la Administración. Estos profesores, y otros, como Jean Château y Jean Stoetzel, venían manteniendo reuniones informales en la Sorbona para discutir las posibilidades de recuperar estos estudios en las universidades francesas, ausentes, excepto algunas iniciativas puntuales, desde el estallido de los años 20 y 30 (Mialaret, 2000, 15-16) ${ }^{3}$. Los representantes del Ministerio propusieron el nombre de pedagogía,

1. A estos dos valores habría que añadir, en una fórmula más amplia, lo sucedido con anterioridad a 1969, cuando el referente era la pedagogía unitaria y general de inspiración alemana. No obstante aquí nos vamos a limitar a los últimos cuarenta años, en los que han predominado los referentes francés y británico.

2. Este artículo forma parte del proyecto de investigación Origen y Desarrollo del conocimiento teórico de la Educación en España, en el que trabajan sus autores. Agradecemos al personal de la Maison de la Recherche de l'Université Paris-Sorbonne, de la Bibliothèque du Centre Universitaire des Saints-Pères y del Institute of Education, University of London, las facilidades prestadas para poder acceder a alguna documentación empleada. No abordamos aquí en extenso el contexto nacional de la evolución de la Teoría de la Educación, que ha sido objeto de otros trabajos. Véase, especialmente, JOVer $(2001,2006)$ y GIL y Jover (2006).

3. Sobre las causas que llevaron a esta ausencia de los estudios de pedagogía en las universidades francesas, escribe Jürgen Schriewer: "Es, pues, la conjunción (i) de la desintegración de la coalición de discursos forjada bajo la República Triunfante, (ii) de los intereses corporativos particulares que hacen prevalecer las disciplinas reunidas en las Facultades de Letras y (iii) de las subsiguientes tendencias a la jerarquización y a la fragmentación disciplinaria, la que hace estallar, en el transcurso de los años 20 y 30, esa creación intelectualmente vulnerable, institucionalizada a partir de 1883-1884 [...] Las únicas formas institucionales, en cuyo seno subsistirá la enseñanza de la pedagogía tras el estallido de la 
pero los miembros de la comisión lograron imponer el de ciencias de la educación (Altet y Mosconi, 2001, 12).

Los nuevos estudios quedaron regulados por una disposición de dos de febrero de 1967, que instituyó cuatro certificados de segundo ciclo en ciencias de la educación, dos de license y otros dos de maîtrise $e^{4}$. Los comienzos fueron modestos, y sólo tres universidades fueron encargadas de organizar inicialmente estos estudios: París, con Debesse, Burdeos, con Château y Wittwer, y Caen, con Mialaret. Más tarde se sumarían otras. La prudencia estaba justificada. La propuesta de creación de estos estudios encontraba fuertes detractores, tanto por parte de algunos miembros de la universidad, que consideraban la pedagogía un saber sin la suficiente consistencia académica, como por parte de los directores de las Escuelas Normales para la formación de maestros, que rechazaban la intromisión de la universidad en un saber que, a su juicio, les pertenecía y para el que no se requería el sustrato de la investigación científica, bastándole la práctica y la experiencia profesional (Mialaret, 2000, 16-19).

Maurice Debesse, a quien parece deberse la iniciativa de la denominación de ciencias de la educación en la comisión de 1966, explicaba unos años más tarde las razones que le llevaron a formularla:

Si propuse y conseguí que se utilizase la expresión ciencias de la educación en el nivel de las enseñanzas universitarias, preferiblemente a hablar de pedagogía, no era en absoluto por desdén hacia este viejo término, o para sustituirlo, mediante una usurpación ridícula, por un título más pomposo. Fue porque la palabra pedagogía se había hecho doblemente equívoca, resultando al mismo tiempo demasiado limitada y vaga.

Equívoca porque la pedagogía de cualquier enseñanza en el nivel elemental, secundario o superior, designa en francés un aspecto de esta enseñanza, su lado didáctico, por decirlo así -con una palabra que nuestros amigos extranjeros están mucho más dispuestos a utilizar que los franceses [...] Equívoca, también, porque la pedagogía designa hoy tanto la práctica educativa cotidiana como la investigación de carácter científico (Debesse, 1973, 71)5.

disciplina, se vinculan a los Institutos de psicología que ven la luz a partir del periodo entreguerras, o a los escasos Certificados de Estudios Superiores que, sometidos a reiteradas transformaciones, se integran en los programas de las licenciaturas de filosofía, sociología o psicología. Sólo bajo estas formas sustitutorias subsistió la pedagogía durante un largo eclipse hasta su resurgimiento en los años sesenta" (SCHRIEwer, 2000, 263-264).

4. Organisation des enseignements et des examens du deuxième cycle d'enseignement dans les facultés des lettres et sciences humaines (Arrêté du 2 Février 1967), Journal Officiel de la République Française, 11 Février 1967.

5. Debesse se refería ya a esta denominación en su prólogo al libro de Mialaret Nouvelle pédagogie scientifique, publicado por Presses Universitaires de France en 1954. En el prólogo Debesse lamenta el estado en el que se encuentran en ese momento la pedagogía experimental y las ciencias de la educación en la enseñanza universitaria francesa (Debesse, 1954, VII-VIII). 
En la motivación original de la implantación de los estudios con esa denominación, se encontraba, pues, la pretensión de destacar la vertiente de estudio científico de la educación, frente al exclusivismo didáctico y la identificación de lo pedagógico con la práctica experiencial.

No faltaron las críticas a esta pretensión de construcción científica, políticamente neutra, del conocimiento de la educación. Críticas que provenían de las teorías de la reproducción, la pedagogía institucional o el sociopsicoanálisis. En el seno del debate acaecido con ocasión de la celebración en París, en septiembre de 1973, del Congreso L'Apport des Sciences Fondamentales aux Sciences de l'Éducation, organizado por la Asociación Internacional de Ciencias de la Educación ${ }^{6}$, Jacques Ardoino, erigido en portavoz de las corrientes alternativas, abogará por una visión amplia y multidimensional de las ciencias de la educación, capaz de acoger las diversas tendencias de la psicología social, las de carácter experimental lo mismo que las de inspiración terapéutica, con independencia de su estatuto científico, y de originar una orientación realmente pluridisciplinar y la quiebra del "mono-racionalismo" tradicional (Ardoino, 1973).

Muchos vieron en la reimplantación de los estudios bajo esta nueva fórmula la ocasión para una auténtica ruptura y renovación. Pero, más allá de las intenciones de quienes participaron en la comisión fundacional, no todos compartían la misma percepción de lo que había que abandonar y lo que convenía poner en su lugar. A las pocas semanas de publicarse la disposición con la organización de los estudios, Gilles Ferry escribe en L'Éducation nationale un impactante artículo en el que, haciendo una crítica a tres libros recientes de la corriente institucional, escritos por De Peretti (1966), Oury y Vasquez (1967) y Lobrot (1967), decreta la mort de la pédagogie:

La sustitución de la "pedagogía" por las "Ciencias de la educación", si es algo más que una concesión puramente formal al lenguaje anglosajón, significa el abandono de las especulaciones normativas a favor de las investigaciones positivistas [...] Entre el mesianismo pedagógico [...] (que al menos tiene el mérito de ocuparse de la práctica educativa), y el experimentalismo inútil [...] (que tiene al menos el mérito del rigor), el estudio objetivo del hecho educativo concreto está por hacerse (Ferry, 1967, 10).

Ferry incluía dentro de ese "mesianismo pedagógico" los tres libros que criticaba, que se situarían para él dentro de la pedagogía muerta: "La "pedagogía"

6. Gérard Mendel y Christian Vogt, autores de Le manifeste éducative: contestation et socialisme (Mendel y Vogt, 1973a) declinan asistir al Congreso por desacuerdo sobre cuestiones de forma y de fondo. El fundador del sociopsicoanálisis y su colega deploran la pretensión de "hablar de las "Ciencias de la educación" ("Asociación internacional de ciencias de la educación", "Congreso internacional de ciencias de la educación") como entidades políticamente neutras ("las ciencias") aislables de las luchas políticas e ideológicas" (Mendel y Vogt, 1973b, 504). 
está muerta. Las tres obras de las que acabamos de hablar son todavía obras de "pedagogía", en la medida en que proclaman, legitiman y recomiendan a la luz de algún credo" (ibid., 11).

En España, los cultivadores de la Pedagogía General asumieron el reto que suponía el nuevo marco epistemológico de las ciencias de la educación. Muchos de ellos vieron en él la posibilidad de abrir el conocimiento pedagógico al mundo de las aportaciones científicas y acercarlo más a la práctica. En La Ciencia de la Educación ¿Pedagogos para qué? García Carrasco (1983, 73-74) se refería todavía a comienzos de los años ochenta a esta doble limitación de la pedagogía española, demasiado aferrada, a su juicio, a una indagación solitaria sobre las cuestiones que tienen que ver con la esencia y las finalidades últimas de la educación. El enfoque de las ciencias de la educación representaba una ocasión para la renovación del campo de conocimiento. Dentro de su esquema pluridisciplinar, la Pedagogía General se reservaba un doble papel. Por una parte, una función epistemológica de reflexión acerca de la naturaleza del saber pedagógico y, por otra, una función de síntesis de las aportaciones, necesariamente parciales, de las diferentes ciencias de la educación, orientada a la generación de principios o normas de acción. Se ponían con ello las bases para la conversión de la Pedagogía de inspiración teleológica en una Teoría de la Educación de carácter más práctico, en sentido científico-tecnológico, que actuase como una especie de guardián omnicomprensivo del conocimiento pedagógico ${ }^{7}$.

De las dos funciones trazadas en el programa original, la Teoría de la Educación, concedió, al menos en los momentos iniciales y como corresponde, quizás, a todo conocimiento joven, mucha más importancia a la primera, esto es, a la justificación de sí misma, mientras que apenas logró adentrarse en la segunda, o al menos no lo hizo en la medida y forma deseada. Hace unos años, Jaume Sarramona hacía balance y reconocía con cierto desencanto:

Explicaciones históricas y pragmáticas aparte, el caso es que el cambio de denominación de "Pedagogía" por "Teoría de la Educación" no ha resuelto las expectativas de quienes lo promovieron, porque el deseo de buscar una fundamentación más empírica y no exclusivamente filosófica a la actuación educativa, que se reservaría para la Filosofía de la Educación, se ha visto condicionada por

7. En el sistema de las ciencias de la educación, escribía José Luis Castillejo en 1976, la Pedagogía General se configura "a modo de atalaya que permite, contemplando todo el ámbito de la Educación, reflexionar analítica y sintéticamente, tras recoger las aportaciones de cada una de las Ciencias e integrarlas en un todo sobre los principios pedagógicos que rigen todo tipo de educación. Al tiempo, le permite ser la única capaz de sistematizar las ciencias de la educación. Precisamente en este sentido se habla de Sistemática y de Pedagogía Fundamental. Se convierte así en una Teoría de la Educación que abarca tanto la vertiente especulativa o teorética fundamental y necesaria a toda ciencia, como la normativa, común a toda ciencia del ámbito educativo; es decir, dirigida en última instancia a la práctica" (CAstillejo, 1976, 137). 
la dinámica corporativista de los docentes universitarios, que ha llevado a calificar a todas las materias abarcadas en el Área de Teoría e Historia de la educación como de meramente especulativas, sin favorecer con ello la vinculación de la teoría con la práctica (Sarramona, 2000, 8).

Ya a comienzos de los ochenta aparecen, desde el entorno de la Teoría de la Educación, las muestras de escepticismo sobre el modo en que se está aplicando el nuevo marco y los intereses que mueven a los cultivadores de las ciencias de la educación (García Carrasco, 1983, 75-76). Sin negar la presión que los intereses y las dinámicas corporativistas hayan podido ejercer en la forma en la que se ha desarrollado la Teoría de la Educación, conviene, a nuestro juicio, preguntarse hasta qué punto esa sensación de haber fracasado en el logro de las expectativas previstas no tiene también una de sus posibles causas en la dificultad de transferencia de los modelos epistemológicos de una tradición y contexto a otro.

En agosto de 2008 tuvimos ocasión de mantener una entrevista en París con uno de los inspiradores de la introducción de las ciencias de la educación en Francia, Gaston Mialaret. Sobre la intención que, desde su punto de vista, latía en esta institucionalización nos comentaba:

La cuestión, para mí, es de giro epistemológico. La pedagogía dice: hay que hacer esto, esto está bien, esto no está bien. Pero es una especie de disciplina prescriptiva, prescribe cosas. Nosotros, las ciencias de la educación, consideramos el hecho educativo como un hecho social y lo examinamos independientemente de las condiciones pedagógicas. He desarrollado aquí la noción de situación de educación [...] Es el momento en el que tiene lugar la acción, que existe, que se produce ahí siempre. Nos separamos de la acción, tomamos distancia para examinar sus razones, no en función de ideas pedagógicas o filosóficas, sino en función de una mirada científica, objetiva [...] En Francia, y es la propia historia de la educación quien nos lo enseña, la pedagogía era dominio de las Escuelas Normales primarias, que formaban a los profesores de escuela primaria. Era ahí donde se aprendía cómo dar la clase. Eso era la pedagogía [...] Y como me gusta repetir a veces, citando a Alfred Binet, en pedagogía todo se ha dicho pero nada se ha probado (G. Mialaret: entrevista, París, agosto de 2008).

Tanto en Francia como en España, las ciencias de la educación se proponen como una alternativa a la pedagogía. Pero lo que el término pedagogía evoca en un sitio y en el otro tiene matices diferentes, y, por tanto, también los adquiere el sentido de la alternativa. En España, que arrastra una institucionalización previa, las ciencias de la educación pretenden ser una alternativa a la vieja pedagogía especulativa que se estudiaba en las universidades, una alternativa que aspiraba a levantarse sobre el modelo de la ciencia experimental. Cuando en España se dice que con las ciencias de la educación se pretende acercar más el conocimiento a la práctica, lo que se quiere subrayar es la necesidad de una pedagogía más a ras de tierra, menos metafísica. 
En Francia, que parte de un vacío institucional prolongado, con la denominación de ciencias de la educación se intentaba, sobre todo, destacar la posibilidad de estudio científico de la educación, más allá de su significado puramente práctico. Dicho de otro modo, se buscaba reintegrar la pedagogía en la universidad bajo un nuevo ropaje que la hiciese más atractiva, lo que exigía diferenciarla de la pedagogía de intención didáctica que se estudiaba en las Escuelas Normales, en la que "todo se ha dicho, pero nada se ha probado". Las ciencias de la educación representaban un tournant épistémologique, con el que el epicentro se movía de la intención prescriptiva sobre el acto educativo, al estudio científico, objetivo y desinteresado, de las situaciones y variables que afectan a su desarrollo. La educación deja de ser un desempeño a realizar, para convertirse en un hecho a estudiar.

En España, la apelación a las ciencias de la educación desde el ámbito de la Teoría de la Educación adquiere un matiz más prescriptivo, en el sentido de que en ellas se busca un fundamento de regulación técnica. En ambos casos se trata, sin embargo, de un conocimiento de la acción y para la acción, pero no desde la acción.

Tampoco las circunstancias de la implantación de las ciencias de la educación fueron las mismas en Francia y en España. En la entrevista que mantuvimos con él, nos comentaba también Mialaret:

El surgimiento en Francia de las ciencias de la educación está ligado a la situación francesa. Francia no es muy pedagoga. No, no lo es, porque... Nuestro gran filósofo francés, Descartes, nos decía que, cuando surge un asunto difícil, se divide en las partes necesarias para llegar a la evidencia. Entonces ino hace falta la pedagogía! La cuestión es que hay que conocer bien la materia, saber cómo trocearla en pequeñas partes, y con eso basta. En consecuencia, Francia no ha estado nunca muy inclinada hacia la pedagogía [... El El problema es que en Francia la pedagogía se ha considerado siempre, siempre, como podríamos decir con el sentido etimológico de la palabra, algo propio del esclavo. Están los sabios, que lo saben todo, y nosotros no sabemos nada [...] La situación francesa no ha aceptado nunca que la pedagogía se sitúe en el nivel universitario [...] Nosotros pensábamos que había que conocer la pedagogía, efectivamente, pero poniendo el énfasis en la situación educativa, analizándola sobre el plano social, psicológico, demográfico, biológico, etc. Son precisamente estos estudios científicos los que constituyen las ciencias de la educación, pero que suponen la adopción de cierta distancia con respecto a la acción pedagógica. Ésta es la cuestión (G. Mialaret: entrevista, París, agosto de 2008).

Mientras que en Francia las ciencias de la educación venían a llenar un vacío, la France n'est pas très pédagogue, nos decía Mialaret, en España su organización se monta sobre la existencia de una estructura previa que la condiciona. Más que del colmado de un vacío institucional, o de una ruptura epistemológica (la mort de la pédagogie!), en nuestro caso hay que hablar de una superposición de marcos de conocimiento, fomentada por las sucesivas modificaciones de los planes de estudio que se hicieron durante la década de los años setenta. A finales de esa década, 
De la Orden se lamentaba de que la aplicación que se estaba haciendo de la sistemática de las ciencias de la educación reflejase más la situación del conocimiento pedagógico de los años cincuenta que la de los setenta, y decía: "Nos hallamos, pues, en un momento de inestabilidad y superposición de planes de estudio que dificultan seriamente la determinación de la posición que ocuparán las distintas disciplinas específicas en el conjunto de los estudios pedagógicos" (De la Orden Hoz, 1979, 249).

Este escenario de indefinición y superposición, administrativa y epistemológica, tiene su reflejo en el desarrollo disciplinar del conocimiento teórico de la educación (Gil y Jover, 2006). Durante los años setenta y los ochenta, la Teoría de la Educación busca su identidad conviviendo en los planes de estudio con disciplinas del viejo y del nuevo esquema, que la hacen bascular entre la filosofía y la ciencia empírica. A partir de su inclusión, a comienzos de los noventa, como materia troncal de la nueva Licenciatura en Pedagogía, se consolida su situación administrativa como sustituta de la antigua Pedagogía General (con lo que, paradójicamente, la desaparición de la Pedagogía General se consuma en nuestro país con el regreso a la Pedagogía como denominación de los estudios de licenciatura). En este respaldo administrativo se ve la ocasión para reemplazar definitivamente la vieja Pedagogía General, de carácter especulativo, por un nuevo conocimiento teórico, sintetizador, de la educación, cuyo objetivo, escribe Colom, "se centra en mejorar la práctica educativa a través del conocimiento que le propician las ciencias de la educación con sus diversas metodologías" (Colom, 1997, 154).

\section{El REFERENTE BRITÁNICO}

Se asume en nuestro entorno que ese intento de modernización que lleva de la Pedagogía General a la Teoría de la Educación se inspira en el mundo anglosajón, más cercano en este sentido al modelo pluridisciplinar francés, que al unitario de la tradición alemana (Colom, 1992, 12-14). Concretamente, el punto de partida para la adopción de este nombre se sitúa en la publicación en 1980 de la versión española, a cargo del catedrático de Lógica y Filosofía de la Ciencia Miguel Ángel Quintanilla, del libro de Terence W. Moore Introducción a la Teoría de la Educación (Moore, 1980), publicado en el Reino Unido seis años antes (Colom, 2003, 125; Sarramona, 2003, 164). Más tarde se publicaría también en español, aunque en esta ocasión por la editorial Trillas de México, su libro Introducción a la Filosofía de la Educación (Moore, 1987), publicado originalmente en la colección International Library of Philosophy of Education, dirigida por Richard Stanley Peters.

La publicación de estas dos obras de Moore responde a un contexto muy determinado, en cuyo fondo, como él reconoce, sigue latiendo la llamada "revolución en filosofía" (ibid., 14-17) que ejercerá una influencia decisiva en la configuración de la Teoría de la Educación británica. Ya en 1942, Charles D. Hardie se quejaba de que "el presente estado de la teoría de la educación, con sus numerosas doctrinas en conflicto, no puede considerarse en absoluto satisfactorio" y abogaba, 
en consecuencia, por una renovación similar a la operada en el campo de la filosofía por Moore, Broad y Wittgenstein (Hardie, 1968, XIX) ${ }^{8}$. La renovación tardaría en llegar. En la década de los cincuenta, recuerda Dearden, las disciplinas pedagógicas constituidas estaban configuradas fundamentalmente por la psicología y la historia, junto a las que convivía un campo indefinido denominado "Principios de educación" (Dearden, 1984, 37). En el discurso de despedida de su Cátedra en el Instituto de Educación de la Universidad de Londres, pronunciado en junio de 1962, señalaba todavía Louis Arnoud Reid la imprecisión en la que se movía la Teoría de la Educación, a modo de un "abrigo de muchos colores", cuyo intento de síntesis obligaría a sus cultivadores a convertirse necesariamente en "afanados amateurs" en las diferentes ramas que la forman (Reid, 1965, 10-16). Y en la misma época, el sucesor de Reid, Richard Stanley Peters, abogaba por la superación de lo que él llamaba la undifferentiated mush de la Teoría de la Educación, "que tanto ha contribuido a la baja reputación del estudio de la educación en este país” (Peters, 1980, 273)?.

Gracias a iniciativas como las emprendidas por Peters en el Instituto de Educación, a partir de la segunda mitad de los sesenta el estudio teórico de la educación experimentó uno de sus mayores impulsos en el Reino Unido, favorecido también por ciertas medidas oficiales que alentaban el desarrollo de las titulaciones específicas de estudios sobre educación y que la formación no se enfocase exclusivamente hacia la capacitación profesional (Dearden, 1984, 3).

8. Se trata de la tercera reimpresión de la edición americana de esta obra, publicada originalmente en el Reino Unido en 1942 por Cambridge University Press. Por otro lado, el mismo año de 1942, James Ross, antiguo director del Westminster Training College, defendía en Groundwork of Educational Theory la necesidad de una mayor fundamentación filosófica de la teoría de la educación, que justificaba, entre otras razones, en los acontecimientos que se sucedían en Europa (Ross, 1942). El propio Ross menciona en esta obra su deuda con tres autores que son referencia fundamental en la configuración de la Teoría de la Educación británica de la primera mitad del siglo XX: John Adams, Professor of Education de la Universidad de Londres, y autor de The Evolution of Educational Theory, donde mantiene una concepción de la teoría como aspecto racional de la práctica, de la que depende y en la que revierte (Adams, 1912); Percy Nunn, catedrático en la misma Universidad, de cuyo Instituto de Educación fue primer director, así como presidente de la Sección Inglesa de la New Education Fellowship, que en 1920 publicaría el influyente libro Education. Its Data and First Principles (Nunn, 1920), fundamentado en la filosofía y la psicología y fuente de inspiración, entre otros, de Maria Montessori (RöHrs, 1994); y Robert R. Rusk, autor de The Philosophical Bases of Education, para quien la indagación filosófica afecta, no sólo a la finalidad, sino a los diferentes aspectos de la educación: del currículo a los métodos, pasando por la elección de libros de texto (Rusk, 1929, 3. ${ }^{a}$ reimpresión). Resulta interesante observar cómo en estos antecedentes se plantea ya el problema del sentido y unicidad de la Teoría de la Educación. Para Adams "como todos los demás estudios que tienen que ver con lo orgánico, la educación debe depender de la ayuda de un amplio número de ciencias, pero no es menos un estudio por sí mismo, con un rango independiente y propósitos definidos. No es una mera ciencia práctica en el sentido de una aplicación mecánica de los principios extraídos desde fuera. Tiene su propia teoría como su propia práctica" (ADAms, 1912, 4).

9. La referencia procede de la célebre conferencia "Education as Initiation", que Peters pronunció en 1963 en su toma de posesión de la Cátedra, en sustitución de Reid. 
El enfoque predominante en este momento es el de la Teoría de la Educación como un conjunto de disciplinas fundamentales que se aplican a la educación, entre ellas, una Filosofía de la Educación de inspiración analítica. La presunción básica es que estas disciplinas proporcionan los elementos sobre los cuales es posible establecer los principios que han de guiar la práctica educativa. Uno de los exponentes más influyentes de este enfoque fue el matemático y filósofo Paul H. Hirst, colaborador de R. S. Peters. En un famoso artículo publicado en 1963, mantendrá Hirst que la Teoría de la Educación, "(i) es distinguible como todas las demás disciplinas por las cuestiones particulares a las que busca dar respuesta, en este caso las preguntas acerca de cierto grupo de actividades prácticas, y (ii) es dependiente de muchas ramas de conocimiento, incluida la filosofía, la comprensión de las cuales provee así la base para la elaboración de los juicios prácticos" (Hirst, 1963, 57).

El enfoque de las disciplinas aplicadas a la educación es paralelo al modelo francés de las ciencias de la educación, por lo que no debe extrañar el tránsito fluido que se opera en nuestro país de uno a otro en el proceso de construcción de la Teoría de la Educación. No obstante, las peculiaridades propias de cada contexto añadirán nuevas disonancias a ese tránsito. Así, en el Reino Unido la Teoría de la Educación estaba lejos de ser considerada la disciplina unitaria ni, mucho menos, la disciplina científico tecnológica que se pretendía aquí. Sobre la primera de estas aspiraciones, tanto Hirst como Peters defendían que el estudio de la educación debía basarse en un trabajo de colaboración entre los especialistas de disciplinas como la historia, la psicología, la sociología y la filosofía, y consideraban desfasada e inadecuada la pretensión de coordinar estas diferentes disciplinas en una sola. Para Peters, abordar positivamente el problema de la debilidad del conocimiento pedagógico exigía reconocer que "ya que la educación no puede ser nunca una disciplina, un planteamiento que acentúe más la importancia de las disciplinas establecidas podría ser de gran beneficio no sólo para la educación sino para estas disciplinas mismas" (Peters, 1963, 19) ${ }^{10}$. En nuestro caso, la dificultad para renunciar a una Teoría de la Educación unitaria se explica, en el fondo, por la persistencia del influjo de la pedagogía general, que encaja mal en el enfoque de las ciencias de la educación y en el de las disciplinas aplicadas.

Sobre la aspiración a convertir la Teoría de la Educación en un conocimiento científico-tecnológico, no puede pasarse por alto la discusión que desde la segunda mitad de los cincuenta venían manteniendo en el Reino Unido Daniel J. O'Connor y Paul H. Hirst ${ }^{11}$. Para el primero, el término "teoría" se refiere a un

10. Desde el otro lado del Atlántico, Israel Scheffler mantendrá en este mismo libro una opinión similar (SCHEFFLER, 1963).

11. La discusión comenzó en 1957 con la publicación del libro de O'Connor Introduction to the Philosophy of Education, y tuvo continuidad en diversas publicaciones a lo largo de los años sesenta y setenta, como en la recopilación de LANGFORD y O'ConNor (1973). 
conjunto lógicamente secuenciado de hipótesis contrastadas mediante la observación. Sólo la investigación empírica, levantada sobre el modelo de las ciencias naturales, proporciona a la teoría la base de un conocimiento objetivo libre de creencias y prejuicios. En consecuencia, concluirá O'Connor, "la palabra "teoría" tal como es usada en los ambientes educativos es generalmente un título de cortesía. Su uso está sólo justificado allí donde aplicamos a la práctica de la educación resultados de la psicología y la sociología bien establecidos experimentalmente" (O'Connor, 1967, 110) ${ }^{12}$

Frente a la posición radical de O'Connor, Hirst abogará por una concepción más pluralista, en la que la Teoría de la Educación se entiende como sistema de proposiciones cuya finalidad es orientar la acción educativa a partir de una variedad de "formas de conocimiento", que proporcionan principios racionales para la acción. Como el propio Hirst recordaría más tarde,

[La] discusión con el Profesor O'Connor me reafirmó en la forma de ver la teoría de la educación fundamentalmente como la disciplina que busca desarrollar principios racionales para la práctica educativa. Con esta finalidad, extrae, por supuesto, todo lo que puede del conocimiento teórico disponible en las ciencias sociales. La psicología de la educación y la sociología de la educación son precisamente subáreas de la psicología y la sociología que pueden emplearse en este sentido. Pero también se basa en la historia, la filosofía y otros muchos campos cercanos, todos los cuales son importantes para la formulación y justificación de sus principios racionales. Y si la teoría de la educación es un área compuesta de este tipo, esto refuerza mi convicción de ver su unidad como la de un conjunto consistente de principios relativos a la práctica a la que apunta, y no como una vasta integración teórica de las disciplinas matrices (Hirst, 1983, 5) ${ }^{13}$.

Terence Moore, cuyo libro Educational Theory: An Introduction se adopta en España como referente del cambio de la vieja Pedagogía especulativa por la nueva Teoría de la Educación, se inspiraba en Hirst, y decía, por ejemplo, que "mientras una teoría científica es básicamente descriptiva y explicativa, una teoría educativa no se dedica a explicar cómo es el mundo. Más bien, como ha mantenido P. H. Hirst, su función principal es guiar la práctica educativa" (Moore, 1980, 18). Sobre la aportación de Moore, nos comentaba Hirst en la entrevista que mantuvimos con él en el Instituto de Educación de Londres en agosto de 2009:

12. Se trata de la sexta reimpresión, de 1967, de Introduction to the Philosophy of Education (1957).

13. Wilfred Carr ha criticado a Hirst, como representante de la filosofía analítica de la educación, no poder desprenderse, a pesar de todo, de su raíz positivista, lo que le hace más cercano a O'Connor de lo que pretende y le impide ofrecer una respuesta adecuada al carácter normativo de la educación (CArr, 2004). Véase el debate mantenido por ambos autores en "Philosophy and Education. A Symposium" (Hirst y Carr, 2005). 
Terry era básicamente un profesor, un docente de Filosofía de la Educación. No era un pensador original. Sus dos libros son, esencialmente, desde nuestro punto de vista, libros para los estudiantes y para el uso de los profesores en clase. No son contribuciones originales a la Filosofía de la Educación. Terry era un docente excelente. Punto. Pero en la literatura acerca del desarrollo de la Filosofía de la Educación, no se le menciona. La razón de esto es tan simple como que él nunca pretendió ser otra cosa que un buen maestro de Filosofía de la Educación, y lo fue. En esto tuvo un gran éxito (P. H. Hirst: entrevista, Londres, agosto de 2009).

Cuando el libro de Moore se publica en España, Hirst, su inspirador, había comenzado a alejarse del enfoque de las disciplinas fundamentales aplicadas a la educación, para adoptar una orientación más cercana al conocimiento desde la práctica. En 1983, escribe:

En la educación, como en cualquier otra actividad, llegamos a comprender sus problemas y sus propuestas desde el compromiso con la actividad misma. Hemos de penetrar el lenguaje de la actividad practicándola [...] Desde este punto de vista, la validez de los principios prácticos debe proceder de su abstracción desde la práctica, antes que de cualquier fundamento teórico independiente (Hirst, 1983, 12).

En la entrevista, Hirst nos comentaba algunos de los factores que influyeron en esta evolución de su postura como reflejo de un cambio más general, empezando por la influencia que, desde dentro de la misma filosofía analítica, ejercieron en la Universidad de Oxford algunos seguidores de Wittgenstein:

La cuestión principal que subyacía en esto, inicialmente en la filosofía académica, era un grupo de católicos romanos, no debido a sus posiciones religiosas, sino porque el conjunto de Santo Tomás de Aquino se basa realmente en Aristóteles. Y ellos consideraban que el fundamento de la moral y la ética no era en absoluto toda esa rígida cuestión acerca de las proposiciones, porque Aristóteles mantenía una visión muy diferente acerca de la naturaleza de la moral y la naturaleza de la verdad. Aquí es donde, al final, todo el sistema tan compacto que teníamos en los años setenta y principios de los ochenta se desplegó en un movimiento que fue desarrollándose progresivamente, y que desde la filosofía en general pasó a nuestro propio terreno (P. H. Hirst: entrevista, Londres, agosto de 2009).

En su reciente libro God, Philosophy, Universities. A Selective History of the Catholic Philosophical Tradition, Alasdair MacIntyre se ha referido a este momento de la Universidad de Oxford, en el que destaca la presencia de la filósofa católica Elizabeth Anscombe, que unía al profundo conocimiento de su maestro Wittgenstein, el de filósofos clásicos, como Aristóteles, San Anselmo o Santo Tomás de Aquino:

Anscombe se consideraba a sí misma dentro del grupo de filósofos analíticos, pues entendía que la filosofía analítica se caracterizaba más por los modos de argumentación y por los tipos de cuestiones que se planteaba, que por mantener una posición filosófica particular. En consecuencia, para ella no suponía ninguna 
dificultad ser ambas cosas, una filósofa católica y una filósofa analítica, como tampoco lo era para su marido Peter Geach, como filósofo de lógica y filósofo moral, o para su estudiante Michael Dummett, en el campo de la lógica y la filosofía del lenguaje, ambos, como ella, entre los más distinguidos filósofos analíticos (MacIntyre, 2009, 162).

Anscombe, a cuyos seminarios asistió Hirst durante la época que pasó en el Departamento de Educación de la Universidad de Oxford como profesor de matemáticas (Hirst, 2008, 116) ${ }^{14}$, tuvo una presencia significativa, durante los años ochenta, en los seminarios de filosofía y en conferencias que pronunció en la Universidad de Navarra ${ }^{15}$. El clima de pensamiento que pudo generar este nudo de vinculaciones ayuda a entender que, desde la Teoría de la Educación de orientación más filosófica y filiación católica, se pretendiese también aquí el hermanamiento de la filosofía analítica de la educación, importada de la escuela de Londres, con la filosofía aristotélica ${ }^{16}$. Desde esta orientación, la filosofía analítica se consideraba una aportación fundamental al desarrollo del conocimiento teórico de la educación, pero que había que saber integrar en una concepción normativa más sólida (Ibáñez-Martín, 1989) ${ }^{17}$.

Como en el Reino Unido, también en España estas tendencias se alinearon con determinadas perspectivas curriculares y del trabajo de los profesores, como la de investigación-acción, que desde finales de los años sesenta venían reclamando una mayor vinculación con la práctica. La evolución institucional en un país y en el otro no fue, sin embargo, la misma. En el Reino Unido, el giro práctico dio lugar en los años ochenta, al predominio de los campos híbridos, como los estudios curriculares y de administración, muy centrados directamente en la acción, y a la progresiva marginación, a partir de la segunda mitad de esa década, de las disciplinas académicas clásicas (Hirst, 1998, 21) ${ }^{18}$. En España, durante los ochenta se afianza la presencia institucional de la Teoría de la Educación, impulsada por la creación, en 1982, de lo que con el tiempo se convertiría en el Seminario Interuniversitario de Teoría de la Educación, animado por ese propósito de dotar a la disciplina de un perfil científico y tecnológico, que convive con una Filosofía de la Educación,

14. En este mismo trabajo autobiográfico, Hirst se refiere a los autores que le ayudaron a dar forma a su cambio de enfoque, subrayando especialmente la influencia del propio MacIntyre.

15. Varias de estas conferencias fueron publicadas en el Anuario Filosófico de la Universidad de Navarra, y han sido más tarde recopiladas en Anscombe (2005).

16. Véase, por ejemplo, Altarejos (1983).

17. Uno de los primeros introductores en nuestro país de la corriente analítica de la Filosofía de la Educación fue J. M. Esteve, autor del prólogo de la publicación española de la recopilación Education and the Development of Reason, editada por Dearden, Hirst y Peters (edición española, 1982). Véase también Esteve $(1977,1979)$.

18. Sobre la evolución del enfoque de las disciplinas aplicadas a la educación y la presión de los estudios curriculares por abrirse paso en el entorno de la investigación educativa desde finales de los años sesenta, véase Richardson (2002). 
institucionalmente diferenciada de la anterior, de vocación más valorativa y práxi$c a$, en el sentido ético aristotélico.

El giro práctico de los ochenta no llevó a Hirst a rechazar el valor del conocimiento teórico, el cual proporciona los recursos necesarios para una práctica educativa crítica. Para Hirst, "así como es absurdo emprender un proceso experimental, digamos, en la práctica de la ingeniería, sin el conocimiento de las ciencias fundamentales correspondientes, lo es también pretender desarrollar prácticas educativas ignorando todo lo que puede ser conocido en las disciplinas teóricas estrictas de los estudios sobre educación" (ibid., 19-20) ${ }^{19}$.

Para otros, sin embargo, esta pretensión encierra sólo la ilusión de los viejos momentos de gloria, y el final inevitable del giro práctico en un contexto de postmodernismo epistemológico es la negación de la posibilidad misma de la Teoría de la Educación. Ésta es la tesis del filósofo de la educación británico Wilfred Carr, uno de los inspiradores de la reforma curricular y la nueva concepción del profesor que introdujo en nuestro país la Ley Orgánica de Ordenación General del Sistema Educativo, y que tuvo una importante presencia en los círculos académicos españoles durante el final de los años ochenta y el comienzo de los noventa, tanto en el entorno de la investigación-acción como en el de la Filosofía de la Educación. Carr avanzó ya esa tesis en una ponencia que presentó en el Congreso de la International Network of Philosophers of Education, que se celebró en Madrid en 2004 (Carr, 2005), y la ha desarrollado más tarde en un polémico artículo titulado "Education without Theory" (Carr, 2006). En la entrevista que mantuvimos con él en agosto de 2009, el autor británico se expresaba rotundamente al respecto:

Lo que hemos hecho a lo largo de los últimos 100 años ha sido discutir acerca de qué tipo de conocimiento es la educación, qué tipo de teoría. ¿Es ciencia? ¿Es filosofía e historia? ¿Cómo cambia la práctica? ¿Cambia la práctica? ¿Desarrollamos la teoría en la práctica, o abstraemos la teoría a partir de buenas prácticas?

Pienso que lo que hace la postmodernidad es considerar esas suposiciones básicas y decir que las mismas eran falsas. Y la razón es porque partimos adoptando las premisas de la modernidad cuando suponemos que tiene que haber algo llamado Teoría de la Educación, que puede servir de guía, mejorar la práctica, informar a la práctica, ser la base de la práctica, o cualquier otra expresión similar que podamos usar... A partir de aquí, lo único que hay que hacer es descubrir lo que es, lo que debe ser ese conocimiento que se encapsula en la teoría, y también determinar cuáles son los procesos y los procedimientos de la investigación más adecuados para desarrollar ese tipo de conocimiento. ¿Cuál es la metodología más adecuada en la investigación educativa? Y todos, en Gran Bretaña durante los últimos 100

19. No obstante, en la entrevista que nos concedió, Hirst seguía manteniendo la imposibilidad de una Teoría de la Educación como disciplina unitaria, y sugirió la posibilidad de abandonar esta denominación para un conocimiento que, a su juicio, cabe mejor bajo el nombre de Filosofía de la Educación. 
años, hemos intentado dar respuesta a estas preguntas. Sin embargo, la razón por la que no hemos llegado a responderlas no es porque sea difícil, sino porque es imposible, porque son preguntas sin sentido (W. Carr: entrevista, Chesterfield, agosto de 2009)

\section{Conclusión}

¿Ha llegado, como dice Carr, el momento de dar a la Teoría de la Educación a dignified end? (Carr, 2006, 150). ¿Existen otras alternativas? ¿Qué consecuencias cabe sacar de nuestro itinerario acerca del futuro de la disciplina?

Según Walsh, el recorrido por la evolución de la Teoría de la Educación británica de la segunda mitad del siglo XX permite extraer la conclusión de que gran parte de sus ambigüedades e incertidumbres se deben a la pérdida de referencia de lo educativo. Desde esta perspectiva, critica algunas de las formulaciones que adoptó el giro de los setenta y los ochenta por su excesiva focalización en la práctica, en detrimento de la práctica específicamente educativa. "El problema a considerar es hasta qué punto el papel preponderante dado a la práctica deliberativa pone en peligro la profundidad y amplitud de la teoría. De modo inicial, puede proponerse que el riesgo es minimizado cuando no perdemos de vista que la práctica en cuestión es la educación, y tenemos cuidado de abarcar esta práctica en su extensión total" (Walsh, 1993, 43). Para Walsh, la naturaleza de la Teoría de la Educación exige volver a considerar lo que constituye la "conciencia de su propia unidad" de las diferentes prácticas guiadas por la voluntad de educar, que permite entender todas ellas como manifestaciones de una práctica singular, la educación. «Emprender una determinada práctica como educación es precisamente informarla con este espíritu integral» (ibid., 48).

Algo similar puede concluirse con respecto a la situación en Francia, donde las ciencias de la educación no consiguieron configurarse como el conocimiento articulado que pretendieron sus inspiradores ${ }^{20}$. En su último libro, Mialaret atribuye la causa de esta situación a la vaguedad de la normativa de 1967, que favoreció que cada centro dispusiese de una gran libertad para la organización de los estudios, de modo que unos han primado los aspectos psicológicos (Burdeos), otros los históricos (París), otros se han orientado hacia la psicosociología (Lyon), o

20. En 1976, Mialaret hablaba ya de "la imagen deformada que dan de ellas mismas las ciencias de la educación como consecuencia del desarrollo desigual y sin relación que unas y otras tienen" (Mialaret, 1976, 89). Frente a esta situación, abogará por lo que él mismo define como la formación en una necesaria "cultura general en ciencias de la educación que, si está bien estructurada, permite a un individuo tomar contacto con casi todos los ámbitos de la actividad científica contemporánea y abre las perspectivas sobre el conjunto de los problemas que se plantea el hombre en este fin de milenio" (ibid., 90). En 1991, Mialaret publica una obra titulada Pédagogie Générale, y en ella vuelve a insistir: "La única respuesta posible está en una formación general a la vez teórica, práctica y científica que construirá una "cultura general" y una base sólida" (Mialaret, 1991, 6). 
hacia la investigación científica (Caen), o hacia las didácticas (Aix-en-Provence), o hacia la filosofía de la educación (París V, Lyon, Estrasburgo), o hacia la economía de la educación (Dijon), o hacia la pedagogía (Toulouse). Para Mialaret, esta imprecisión ha tenido un aspecto positivo, pues la variedad de orientaciones enriquece el campo, pero ha originado también un efecto negativo: "Ante la posibilidad de obtener un puesto, algunos presentan su candidatura y enseñan un poco lo que quieren..., a veces muy lejos de las auténticas ciencias de la educación" (Mialaret, 2006, 52). La difuminación del concepto de educación a que ha dado lugar esta disgregación del conocimiento requiere cierto esfuerzo por volver a situar en el centro del discurso su núcleo definitorio, esto es, la idea misma de educación, perdida a lo largo del trasiego.

Volvamos ahora a la famosa anotación de Fermat con la que empezábamos este artículo. ¿Llevaba éste razón cuando anotó que la ecuación $\mathrm{x}^{\mathrm{n}}+\mathrm{y}^{\mathrm{n}}=\mathrm{z}^{\mathrm{n}}$, para todo n mayor de 2, es una ecuación imposible? En otra de sus anotaciones, él mismo ya daba pistas para la demostración en el caso de que el exponente fuese 4. Otros matemáticos fueron demostrándolo a lo largo de años y más años para otros exponentes. Pero por este camino no es posible llegar a una demostración final, porque es irrealizable poder demostrarlo para todos y cada uno de una serie infinita de números, incluso usando las más potentes computadoras modernas. Hacía falta una demostración general. Ésta llegaría en junio de 1993, cuando un joven matemático inglés, Andrew Wiles, pronunció en el Isaac Newton Institute de Cambridge tres conferencias que contenían la demostración del último teorema de Fermat. Tras algunos ajustes y comprobaciones, en mayo de 1995 Annals of Mathematics publicó los trabajos de Wiles (Singh, 1998). El reto matemático más popular de la historia había sido superado. La ecuación $\mathrm{x}^{\mathrm{n}}+\mathrm{y}^{\mathrm{n}}=\mathrm{z}^{\mathrm{n}}$ no tiene soluciones enteras cuando $\mathrm{n}$ es mayor que 2. Es una ecuación imposible.

Los matemáticos tardaron más de trescientos años en demostrar el último teorema de Fermat. Esperemos tardar nosotros algo menos en comprobar que un poco de pädagogik a la alemana, más otro poco de sciences de l'éducation a la francesa, más algo de educational studies a la británica da también una fórmula poco prometedora, por cuanto supone aunar elementos heterogéneos desgajados de sus contextos de origen, en los que intervienen situaciones institucionales específicas, coyunturas político-sociales y tradiciones intelectuales diversas, selecciones y exclusiones epistemológicas que priman unas opciones en detrimento de otras, etc. (Schriewer, 2000, 233). En un entorno de internacionalización del conocimiento, sobre todo si hablamos de la universidad, no se trata, claro, de desdeñar provincianamente las aportaciones que puedan venir de otros contextos, sino de enfatizar que para resolver la ecuación de la educación lo que necesitamos es una teoría centrada en investigar la educación misma, como una práctica singular que exige aunar dos exigencias contradictorias: la apelación a una imagen directiva, moral, del ser humano educable y la atención a las condiciones contingentes en que se realiza la acción, y de las que participa la propia teoría, en sí misma una práctica situada. 


\section{REFERENCIAS BIBLIOGRÁFICAS}

Adams, J. (1912) The evolution of Educational Theory. London, MacMillan.

Altarejos, F. (1983) Educación y felicidad. Pamplona, Eunsa.

Altet, M. y Mosconi, N. (dirs.) (2001) Les sciences de l'éducation: enjeux, finalités et défis. Paris, AECSE-INRP.

Anscombe, G. E. M. (2005) La filosofía analitica y la espiritualidad del hombre. Lecciones en la Universidad de Navarra. Pamplona, Eunsa.

Ardoino, J. (1973) Texte d'orientation, en L'Apport des Sciences Fondamentales aux Sciences de l'Éducation, vol. 2. Paris, Éditions de l'Épi, 417-430.

CARR, W. (2004) Philosophy and Education. Journal of Philosophy of Education, 38 (1), 55-73.

- (2005) The role of theory in the professional development of an educational theorist. Pedagogy, Culture \& Society, 13 (3), 333-346.

- (2006) Education without Theory. British Journal of Educational Studies, 54 (2), 136159.

Castillejo, J. L. (1976) Nuevas perspectivas en las ciencias de la educación. I Pedagogía General. Madrid, Anaya.

Colom, A. J. (1992) El saber de la Teoría de la Educación. Su ubicación conceptual. Teoría de la Educación. Revista Interuniversitaria, 4, 11-19.

- (1997) La Teoría de la Educación: contexto actual de los estudios pedagógicos, en CoLOM, A. J. (coord.) Teorias e instituciones contemporáneas de la educación. Barcelona, Ariel, 145-155.

- (2003) Lo político y lo comunitario en la teoría de la educación española: una revisión, en Ortega, P. (ed.) Teoría de la Educación, ayer y hoy. Murcia, Selegráfica, 109-158.

De la Orden Hoz, A. (1979) Enseñanza superior de la Pedagogía y formación del profesorado. La experiencia española, en Escolano, A. (comp.) Los estudios de ciencias de la educación: currículum y profesiones. Salamanca, Ediciones de la Universidad de Salamanca, Instituto de Ciencias de la Educación, 243-252.

De PeretTI, A. (1966) Liberté et relations humaines ou l'inspiration non-directive. Paris, Éditions de l'Épi.

DeARDEn, R. F. (1984) Theory and practice in education. London, Routledge and Kegal Paul.

Dearden, R. F.; Hirst, P. H. y Peters, R. S. (1982) Educación y desarrollo de la razón: formación del sentido crítico. Madrid, Narcea.

Debesse, M. (1954) Préface, en Mialaret, G. Nouvelle pédagogie scientifique. Paris, PUF, VIIXII.

- (1973) Défi aux Sciences de l'Éducation, en L'Apport des Sciences Fondamentales aux Sciences de l'Éducation, vol. 1. Paris, Éditions de l'Épi, 70-78.

Esteve, J. M. (1977) Autoridad, obediencia y educación. Madrid, Narcea.

- (1979) Lenguaje educativo y teorías pedagógicas. Madrid, Anaya.

Ferry, G. (1967) Mort de la pédagogie. L'Éducation nationale, 23 (820), 9-11.

García Carrasco, J. (1983) La Ciencia de la Educación. Pedagogos ¿para qué? Madrid, Santillana.

Gil, F. y Jover, G. (2006) Memoria y prospectiva de la Teoría de la Educación. Revista Portuguesa de Pedagogía, 40 (2), 204-226.

Hardie, C. D. (1968) Truth and Fallacy in Educational Theory. New York, Teachers College Press. 
Hirst, P. H. (1963) Philosophy and Educational Theory. British Journal of Educational Studies, 12 (1), 51-64.

- (1983) Educational theory, en HisRst, P. H. (ed.) Educational theory and its foundation disciplines. London, Routledge and Kegan Paul, 3-29.

- (1998) Philosophy of Education: The evolution of a Discipline, en Haydon, G. (ed.) 50 Years of Philosophy of Education. London, University of London, Institute of Education, $1-22$.

- (2008) In pursuit of reason, en WaKs, L. J. (ed.) Leaders in Philosophy of Education. Intellectual Self Portraits. Rotterdam, Sense Publishers, 113-124.

Hirst, P. H. y CARr, W. (2005) Philosophy and Education. A Symposium. Journal of Philosophy of Education, 39 (4), 615-632.

IbáÑ̃z-Martín, J. A. (1989) El concepto y las funciones de una Filosofía de la Educación a la altura de nuestro tiempo, en Altarejos, F. et al. Filosofía de la Educación hoy. Conceptos, autores, temas. Madrid, Dykinson, 409-419.

Jover, G. (2001) Philosophy of Education in Spain at the Threshold of the $21^{\text {st }}$ Century. Origins, Political Contexts, and Prospects, Studies in Philosophy and Education, 20 (4), 361-385.

- (2006) La construcció disciplinària del coneixement teòric de l'educació: crònica de discontinuïtats en tres actes i epíleg., Temps d'Educació, 31, 85-102.

Langford, G. y O'Connor, D. J. (eds.) (1973) New Essays in the Philosophy of Education. London, Routledge and Kegal Paul.

Lовrot, M. (1967) La pédagogie institutionnelle. L'école vers l'autogestion. Paris, Gauthier.

MacIntyre, A. (2009) God, Philosophy, Universities: A Selective History of the Catholic Philosophical Tradition. London, Continuum.

Mendel, G. y Vogt, C. (1973a) Le manifeste éducative: contestation et socialisme. Paris, Payot, 1973.

- (1973b) Lettre ouverte à la commission "Psychologie Social et Nouvelles Approches Pédagogiques" du Congrès International des Sciences de l'Éducation, en L'Apport des Sciences Fondamentales aux Sciences de l'Éducation, vol. 2. Paris, Éditions de l'Épi, 504.

Mialaret, G. (1976) Les sciences de l'éducation. Paris, PUF.

- (1991) Pédagogie Générale. Paris, PUF.

- (2000) La création des sciences de l'éducation, en Froment, M.; Caillot, M. y Roger, M. (dirs.) 30 ans de sciences de l'éducation à Paris V. Paris, PUF, 15-22.

- (2006) Sciences de l'éducation. Paris, PUF.

Moore, T. W. (1980) Introducción a la Teoría de la Educación. Madrid, Alianza.

- (1987) Introducción a la Filosofía de la Educación. México, Trillas.

Nunn, P. (1920) Education. Its Data and First Principles. London, Edward Arnold.

O'Connor, D. J. (1967) Introduction to the Philosophy of Education. London, Routledge and Kegal Paul.

Oury, F. y Vasquez, A. (1967) Vers une pédagogie institutionnelle. Paris, Maspero.

Peters, R. S. (1963) Comments to J. Walton: A Discipline of Education, en Walton, J. y Kuethe, J. L. (eds.) The Discipline of Education. Madison, The University of Wisconsin Press, $17-22$.

- (1980) Education as initiation, en Gordon, P. (ed.) The study of education: a collection of inaugural lectures, vol. I. London, Woburn Press, 273-299.

Redondo, E. (1972) Presentación, en Tusquets, J. Teoría de la Educación. Madrid, Magisterio Español, 9-11. 
ReID, L. A. (1965) Philosophy and the Theory and Practice of Education. London, Evans Brothers.

Richardson, W. (2002) Educational Studies in the United Kingdom, 1940-2002. British Journal of Educational Studies, 50 (1), 3-56.

RöHRs, H. (1994) María Montessori. Prospects, XXIV (1/2), 169-183.

Ross, J. S. (1942) Groundwork of Educational Theory. London, George G. Harrap.

Rusk, R. R. (1929) The Philosophical Bases of Education. London, University of London Press.

Sarramona, J. (2000) Teoría de la Educación. Reflexión y normativa pedagógica. Barcelona, Ariel.

- (2003) La perspectiva tecnológica en la acción educativa, en Ortega, P. (ed.) Teoría de la Educación, ayer y boy. Murcia, Selegráfica, 159-204.

Scheffler, I. (1963) Is Education a Discipline?, en Walton, J. y Kuethe, J. L. (eds.) The Discipline of Education. Madison, The University of Wisconsin Press, 47-61.

- (2000) Estudios multidisciplinares y reflexiones filosófico-hermenéuticas: la estructuración del discurso pedagógico en Francia y Alemania, en Ruzz BerRIo, J. (ed.) La cultura escolar de Europa. Tendencias históricas emergentes. Madrid, Biblioteca Nueva, 231-269.

SingH, S. (1998) El enigma de Fermat. La historia de un teorema que intrigó durante más de trescientos años a los mejores cerebros del mundo. Barcelona, Planeta.

Torrecillas Jover, B. (1999) Fermat. El mago de los números. Madrid, Nivela.

Walsh, P. (1993) Education and Meaning: Philosophy in Practice. London, Cassell. 